\title{
Elusive seed formation via electrical confinement: control of a novel co-crystal in cooling crystallization
}

\author{
Aneesa J Al-Ani ${ }^{1,2}$, Posy Sugden ${ }^{2}$, Chick C Wilson ${ }^{1,2}$, Bernardo Castro-Dominguez ${ }^{1,31}$ \\ ${ }^{1}$ Centre for Sustainable and Circular Technologies (CSCT), University of Bath, Claverton Down, \\ Bath BA2 7AY, United Kingdom. \\ 2 Department of Chemistry, University of Bath, Claverton Down, Bath BA2 7AY, United Kingdom. \\ ${ }^{3}$ Centre for Advanced Separations Engineering, Department of Chemical Engineering, University of \\ Bath, Claverton Down, Bath BA2 7AY, United Kingdom.
}

\section{$\underline{\text { Supporting information (SI) }}$}

SI Table 1. The experimental data set for electrospray crystallizations in IPA: $\mathrm{H}_{2} \mathrm{O}(40: 60)$.

\begin{tabular}{|c|c|c|c|c|c|}
\hline Experiment & $\begin{array}{c}\text { Weight } \\
\text { percentage } \\
\text { MCM (\%) }\end{array}$ & $\begin{array}{c}\text { Weight } \\
\text { percentage } \\
\text { PCM (\%) }\end{array}$ & $\begin{array}{c}\text { Flow rate } \\
\left(\mathrm{mL} \mathrm{h}^{-1}\right)\end{array}$ & $\begin{array}{c}\text { Electric field } \\
\left(\mathrm{kV} \mathrm{cm}^{-1}\right)\end{array}$ & $\begin{array}{c}\text { Product } \\
\text { obtained }\end{array}$ \\
\hline E1 & 100 & 0 & 0.5 & 1.30 & MCM form I \\
\hline E2 & 90 & 10 & 0.5 & 1.30 & MCM form I \\
\hline E3 & 75 & 25 & 0.5 & 1.30 & $\begin{array}{c}\text { MCM form I } \\
\text { and MCM PCM } \\
\text { co-crystal }\end{array}$ \\
\hline E4 & 50 & 50 & 0.5 & 1.30 & $\begin{array}{c}\text { MCM PCM co- } \\
\text { crystal }\end{array}$ \\
\hline E5 & 25 & 75 & 0.5 & 1.30 & PCM form I \\
\hline E6 & 10 & 90 & 0.5 & 1.30 & PCM form I \\
\hline E7 & 0 & 100 & 0.5 & 1.30 & PCM form I \\
\hline E8 & 50 & 50 & 0.15 & 1.30 & PCM form II \\
\hline E9 & 50 & 50 & 0.25 & 1.30 & PCM form I \\
and II \\
\hline E10 & 50 & 50 & 0.5 & 1.25 & PCM form I \\
\hline E11 & 50 & 50 & 0.5 & 1.00 & PCM form I \\
\hline
\end{tabular}

${ }^{*} \mathrm{H}$ NMR analysis revealed that PCM were present in the product obtained for experiment E2 although not observed by PXRD; it was concluded that PCM was amorphous. Similarly, the presence of MCM was confirmed by ${ }^{1} \mathrm{H}$ NMR for experiments E5-E11.

\footnotetext{
${ }^{1}$ Corresponding Author: Phone Number: +44 122538 49461; Email: bcd28@,bath.ac.uk
} 
SI Table 2. Seeded cooling crystallizations.

\begin{tabular}{|c|c|c|c|c|c|c|c|}
\hline Experiment & $\begin{array}{l}\text { Seed size } \\
\quad(\mu \mathrm{m})\end{array}$ & Solvent & $\begin{array}{c}\text { Stirring } \\
\text { (rpm) }\end{array}$ & $\begin{array}{l}\text { Dwell time } \\
\text { at } 4^{\circ} \mathrm{C}\end{array}$ & $\begin{array}{c}\text { Seed } \\
\text { loading } \\
(\%)\end{array}$ & $\begin{array}{l}\text { Product } \\
\text { obtained }\end{array}$ & $\begin{array}{c}\text { Single } \\
\text { crystals } \\
\text { of co- } \\
\text { crystal }\end{array}$ \\
\hline E12 & 150 & $\begin{array}{c}\text { IPA: } \mathrm{H}_{2} \mathrm{O} \\
(4: 6)\end{array}$ & $x$ & $12 \mathrm{~h}$ & 2 & $\begin{array}{l}\text { MCM } \\
\text { form I, } \\
\text { PCM } \\
\text { form I } \\
\end{array}$ & - \\
\hline E13 & 150 & $\begin{array}{c}\text { IPA: } \mathrm{H}_{2} \mathrm{O} \\
(4: 6)\end{array}$ & $x$ & $24 \mathrm{~h}$ & 2 & $\begin{array}{c}\text { MCM } \\
\text { form I, } \\
\text { PCM } \\
\text { form I, } \\
\text { co- } \\
\text { crystal }\end{array}$ & $x$ \\
\hline E14 & 150 & $\begin{array}{c}\text { IPA: } \mathrm{H}_{2} \mathrm{O} \\
(4: 6)\end{array}$ & $x$ & $72 \mathrm{~h}$ & 2 & $\begin{array}{c}\text { co- } \\
\text { crystal }\end{array}$ & $\checkmark$ \\
\hline E15 & 150 & IPA & $x$ & $24 \mathrm{~h}$ & 2 & $\begin{array}{c}\text { no- } \\
\text { product }\end{array}$ & - \\
\hline E16 & 150 & IPA & 700 & $24 \mathrm{~h}$ & 2 & $\begin{array}{c}\text { co- } \\
\text { crystal }\end{array}$ & $x$ \\
\hline E17 & 150 & $\begin{array}{c}\text { IPA: } \mathrm{H}_{2} \mathrm{O} \\
(4: 6)\end{array}$ & $x$ & $72 \mathrm{~h}$ & $\begin{array}{c}0.5,1, \\
2 \& \\
5 \%\end{array}$ & $\begin{array}{c}\text { co- } \\
\text { crystal }\end{array}$ & $\checkmark$ \\
\hline E18 & $\begin{array}{c}25^{*}, 50^{*}, \\
75^{*}, 100 \\
\& 150\end{array}$ & $\begin{array}{c}\text { IPA: } \mathrm{H}_{2} \mathrm{O} \\
(4: 6)\end{array}$ & $x$ & $72 \mathrm{~h}$ & 2 & $\begin{array}{l}\text { co- } \\
\text { crystal }\end{array}$ & $\checkmark$ \\
\hline
\end{tabular}

*trace amount of starting material were observed alongside co-crystal formation.

SI Table 3. The experimental data set for un-seeded crystallizations.

\begin{tabular}{|c|c|c|c|}
\hline Experiment & Crystallization method & $\begin{array}{c}\text { Weight } \\
\text { percentage of } \\
\text { MCM: PCM } \\
(\%)\end{array}$ & Product obtained \\
\hline E19 & Cooling & $50: 50$ & $\begin{array}{c}\text { PCM form I, PCM form II } \\
\text { and MCM form I }\end{array}$ \\
\hline E20 & Evaporative & $50: 50$ & PCM form I and MCM form I \\
\hline E21 & Solution-mediated (slurry) & $50: 50$ & PCM form I and MCM form I \\
\hline E22 & Mechano-chemical (grinding) & $50: 50$ & PCM form I and MCM form I \\
\hline E23 & Vapour diffusion & $50: 50$ & No crystallization \\
\hline E24 & Liquid assisted grinding (IPA) & $50: 50$ & PCM form I and MCM form I \\
\hline E25 & $\begin{array}{c}\text { Liquid assisted grinding (IPA : } \\
\left.\mathrm{H}_{2} \mathrm{O} 4: 6\right) \text { ) }\end{array}$ & $50: 50$ & PCM form I and MCM form I \\
\hline
\end{tabular}

*Experimental methods for each crystallization experiment is available in SI experimental. 


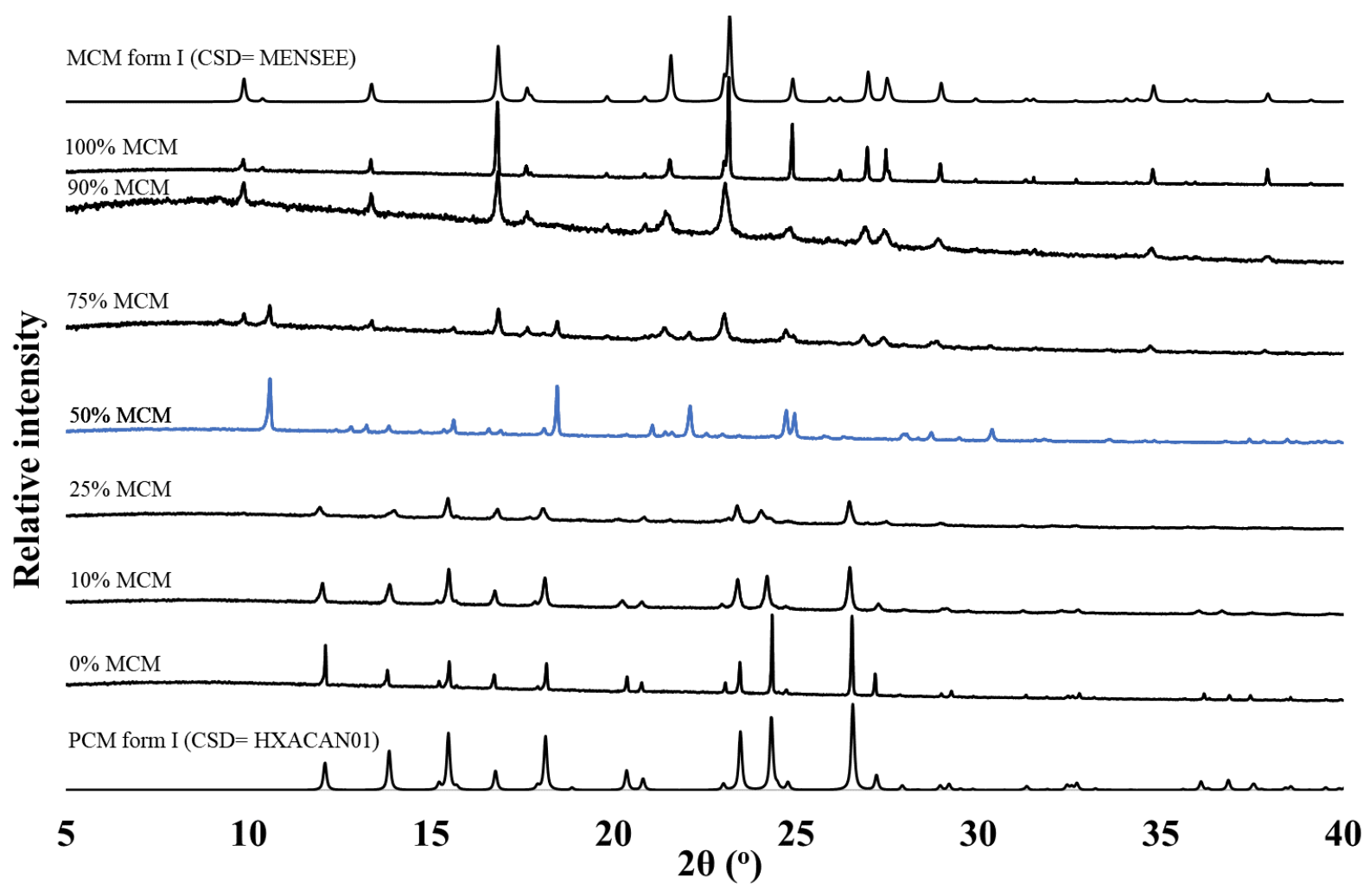

SI Figure 1. The PXRD patterns obtained from experiments E1-E7 in Table 1. The PXRD pattern of the pure MCM PCM co-crystal is highlighted in blue.

Note that the XRD pattern of E2 in SI figure 1 has distinct characteristics compared to the other experimental XRD patterns. The pattern belonging to E2 shows characteristics of a partially amorphous material, with Bragg peaks that are not as well defined and sharp. Further characterization of the bulk material from E2, including DSC and ${ }^{1} \mathrm{H}$ NMR, confirms the presence of both PCM and MCM. Although not well defined, Bragg peaks are observed for MCM in E2, however, no clear Bragg peaks are observed that belong to PCM. MCM appears to be partially amorphous and PCM almost, if not fully amorphous. A comparison of the XRD patterns of E2, E3 and E4 suggests that 1:1 is the optimal stoichiometric ratio of the two components to access and stabilize co-crystal formation (E4). A higher stoichiometric ratio of MCM (75\%) also yielded the co-crystals alongside an access of MCM (E3). E2 contains partially crystalline MCM in addition to amorphous material. It is unknown if this amorphous material is the co-crystal, starting material or possibly a combination of both in an amorphous state. 


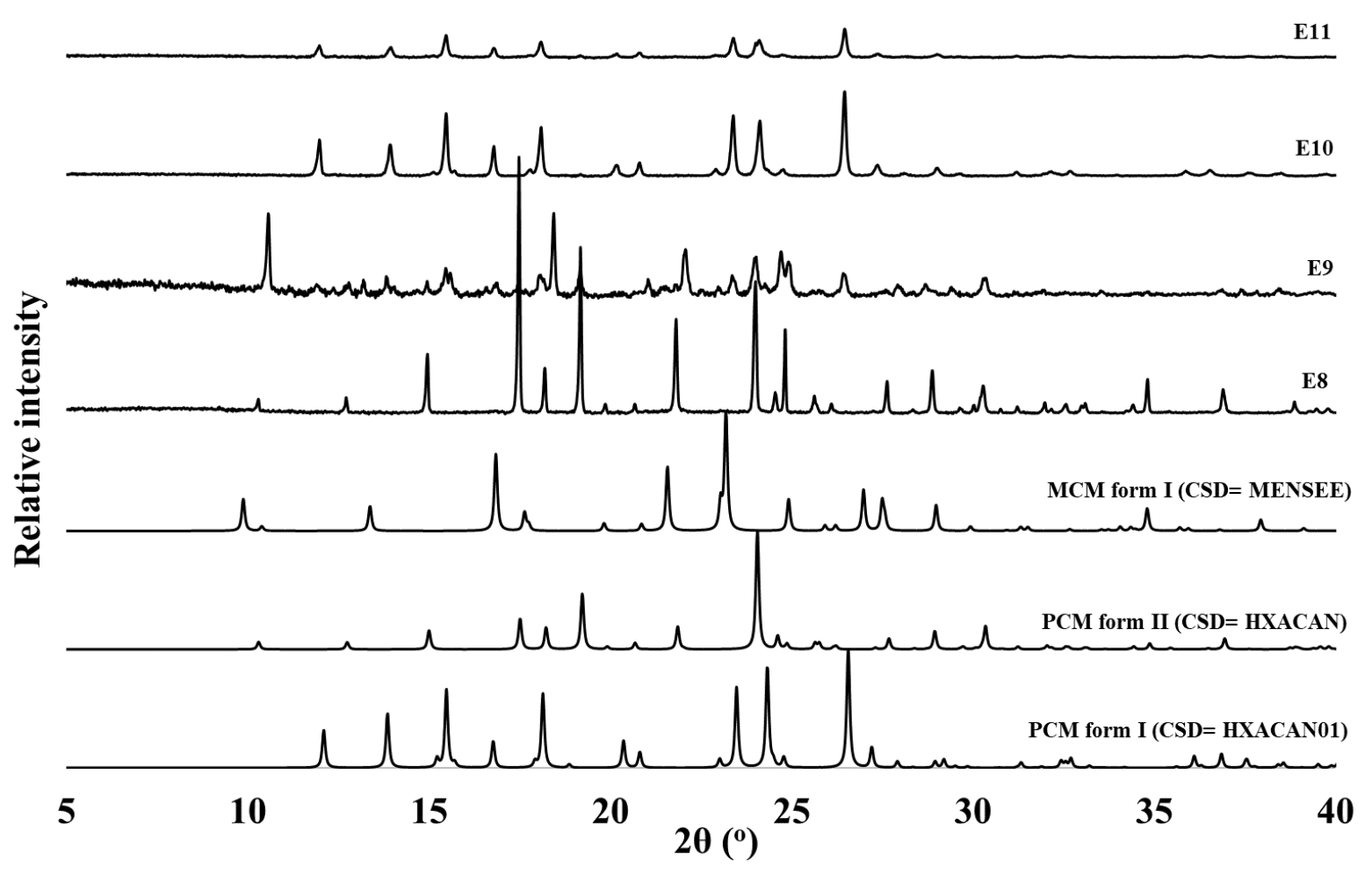

SI Figure 2. The PXRD patterns obtained from experiments E8-E11 in SI Table 1.

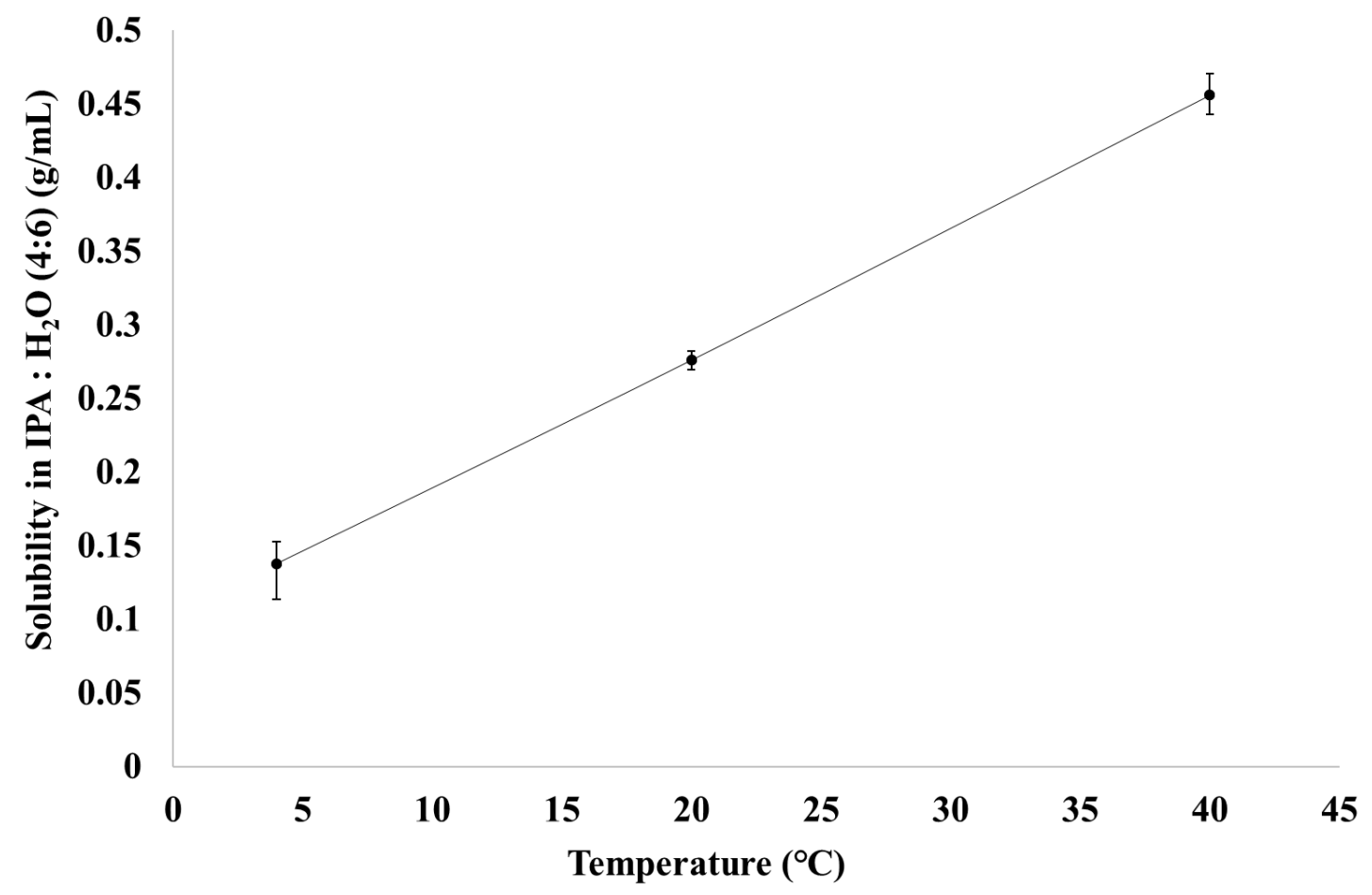

SI Figure 3. The solubility curve for a 1:1 mixture of MCM-form I and PCM-form I in IPA and water (4:6), determined by gravimetric methods. An outline of the method used is available in the SI experimental section. 


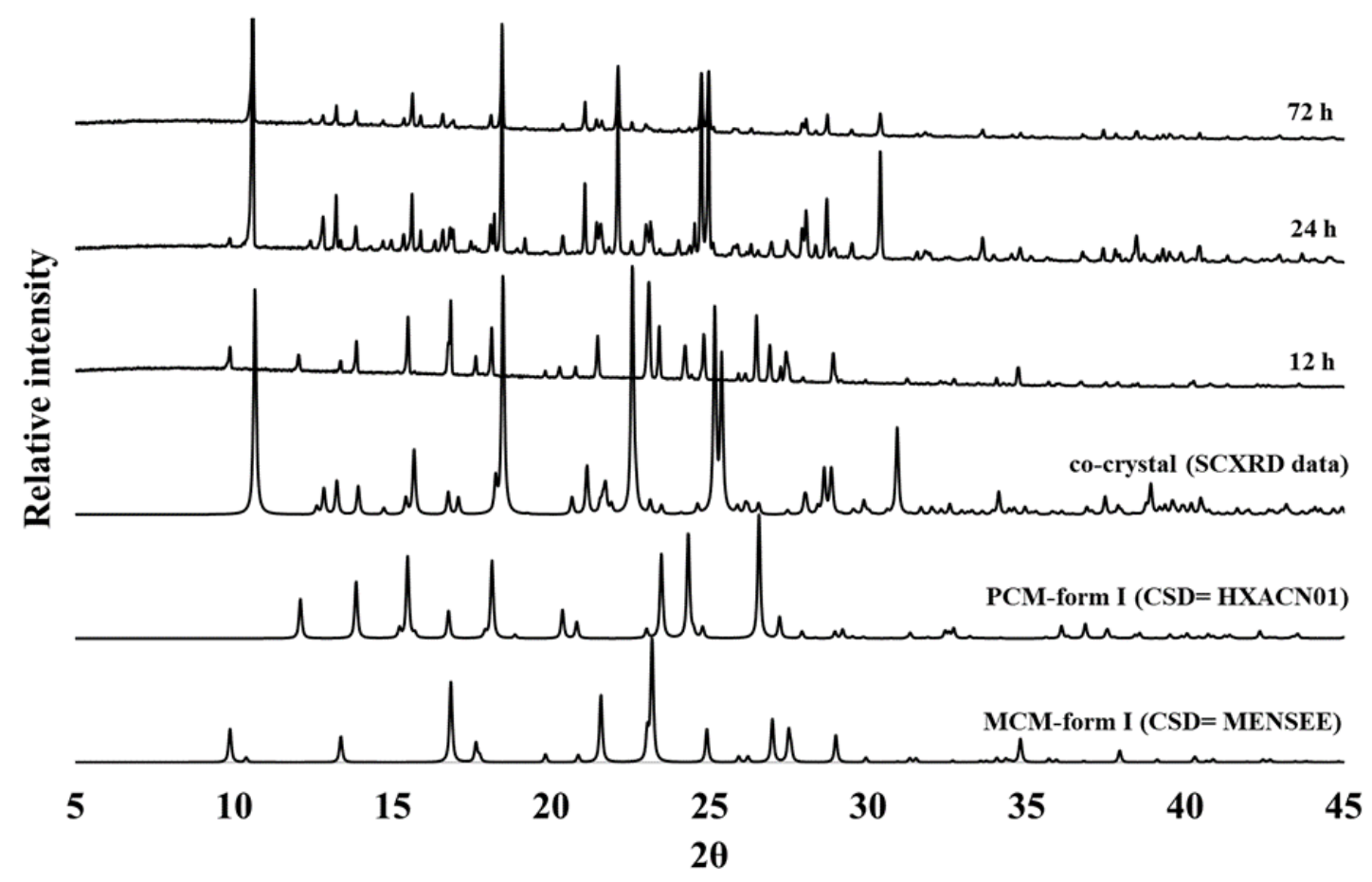

SI Figure 4. Time monitored PXRD traces of co-crystallization under seeded cooling conditions monitored at 12, 24 and $72 \mathrm{~h}$, from experiments E12-14. A 2\% seeded dose of $150 \mu \mathrm{m}$ seeds were used.

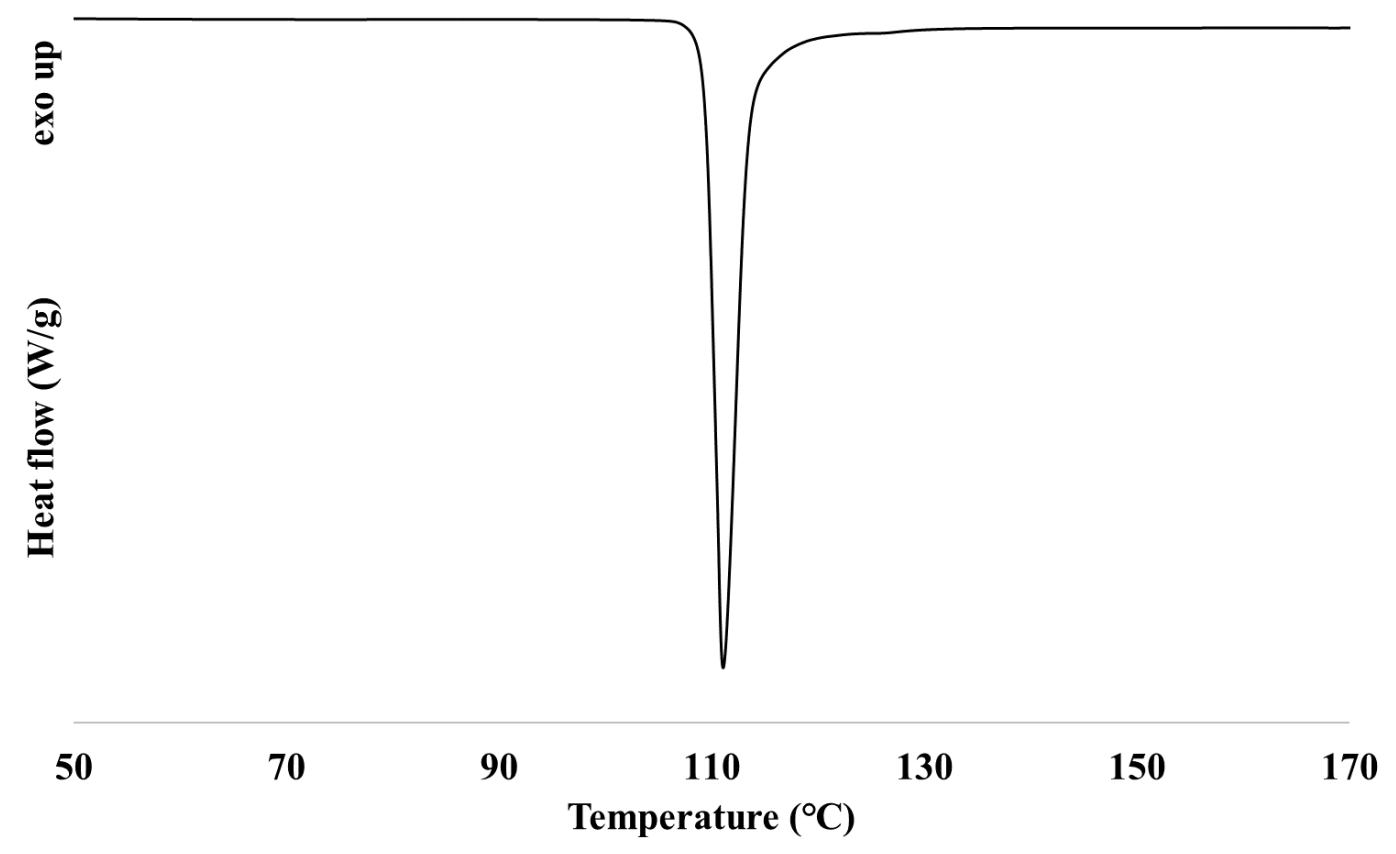

SI Figure 5. The DSC trace obtained for the MCM PCM co-crystal, onset $=104^{\circ} \mathrm{C}$. 


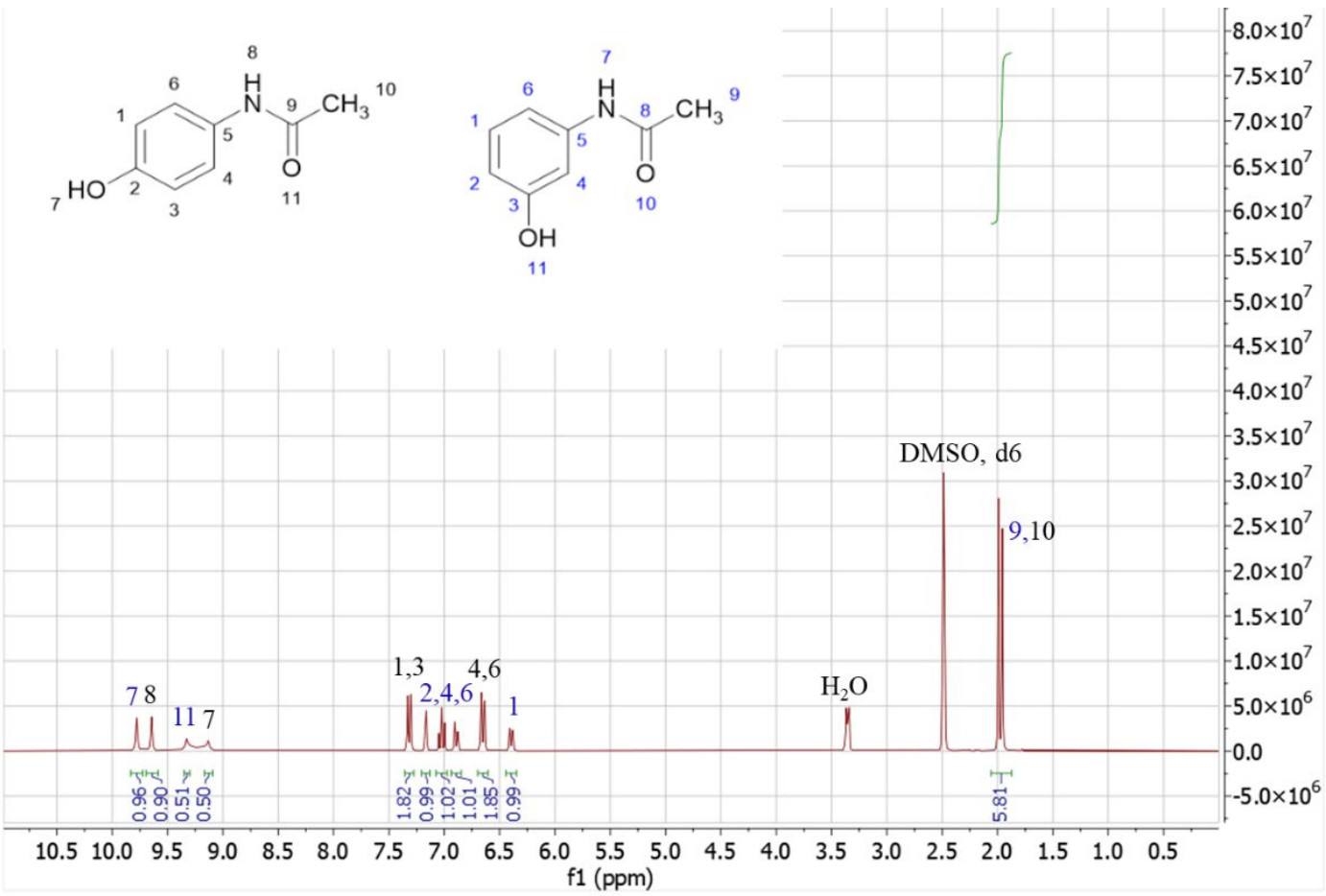

SI Figure 6. The ${ }^{1} \mathrm{H}$ NMR spectrum of the MCM PCM co-crystal which suggests a 1:1 ratio between the multicomponent system.

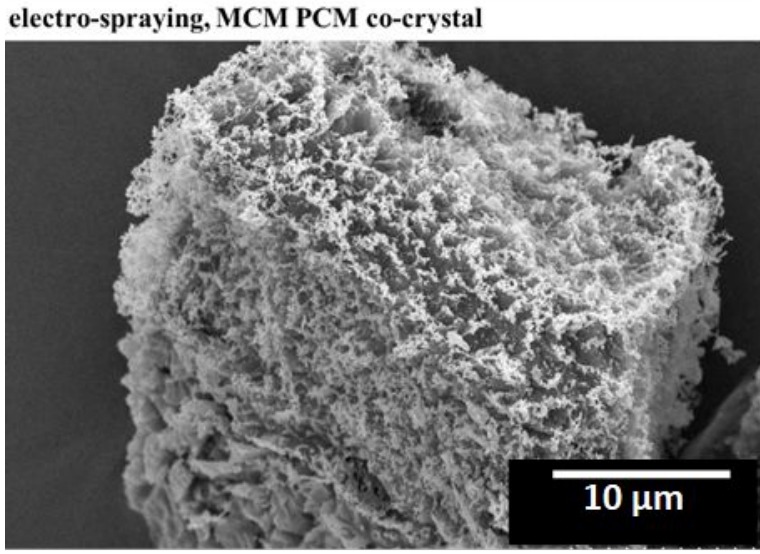

seeded cooling crystallisation, MCM PCM co-crystal

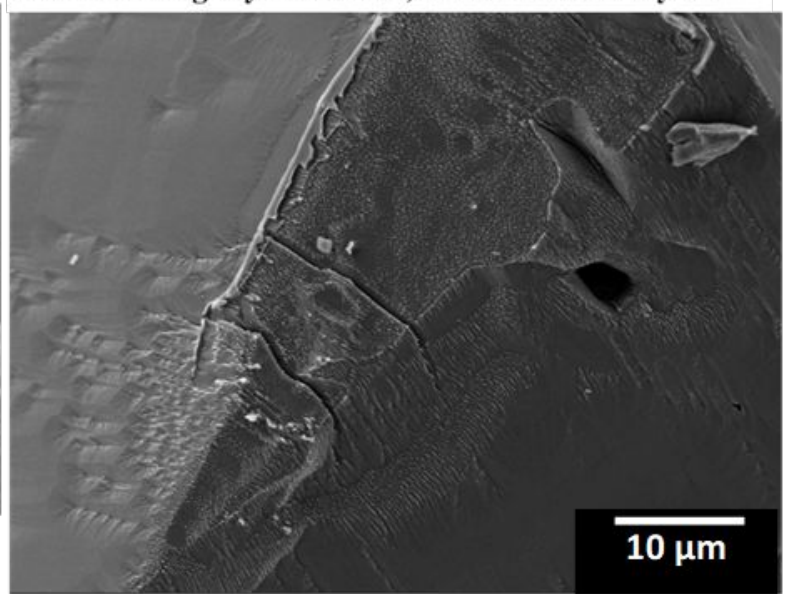

SI Figure 7. A comparison of the surface topography from SEM analysis of the MCM PCM co-crystal product from electro-spraying and seeded cooling crystallization. 


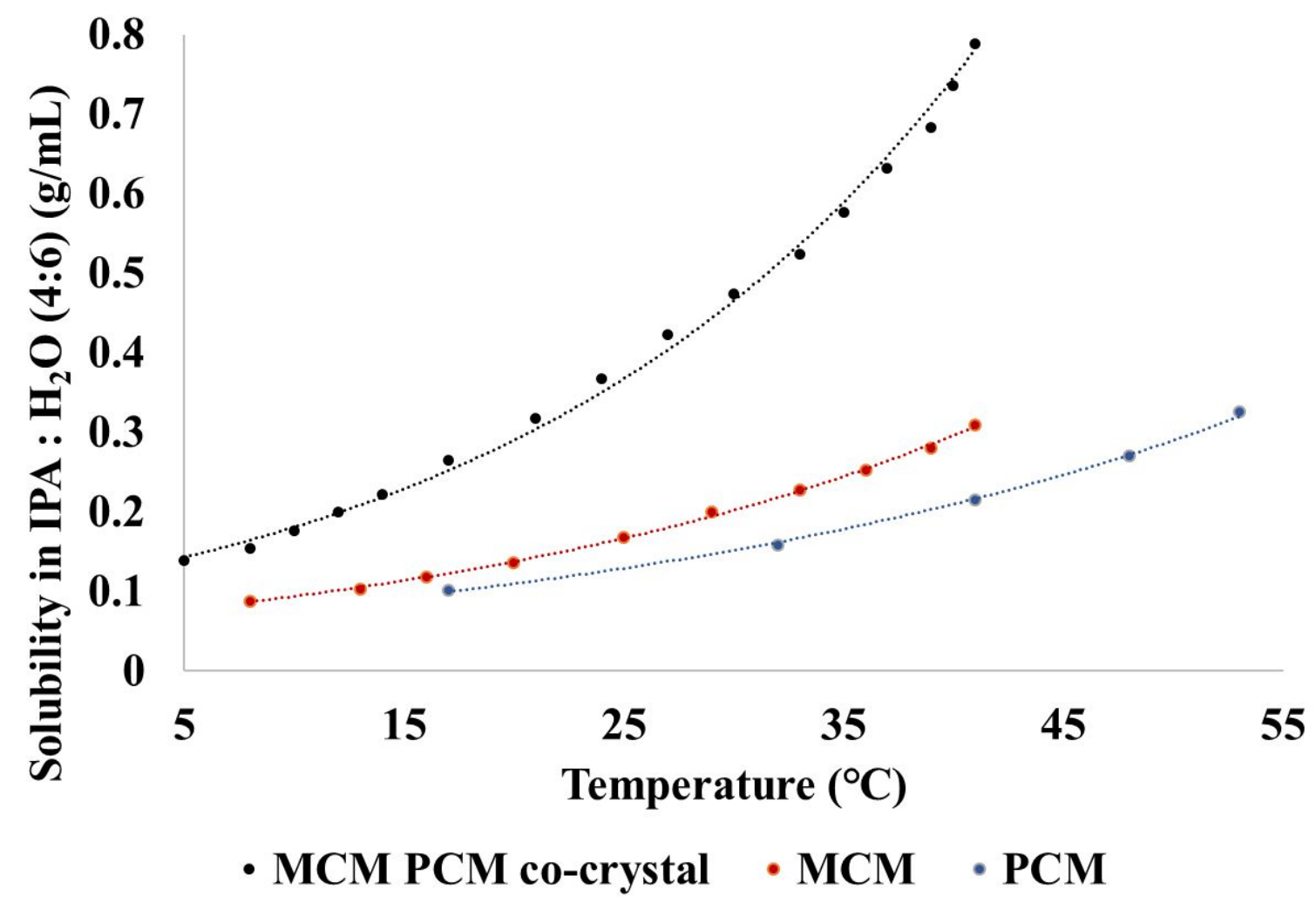

SI Figure 8. The solubility curves of the new co-crystal (black), MCM (red) and PCM (blue) in IPA and water (4:6) determined via observational methods. An outline of the method used is available in the SI experimental section. 


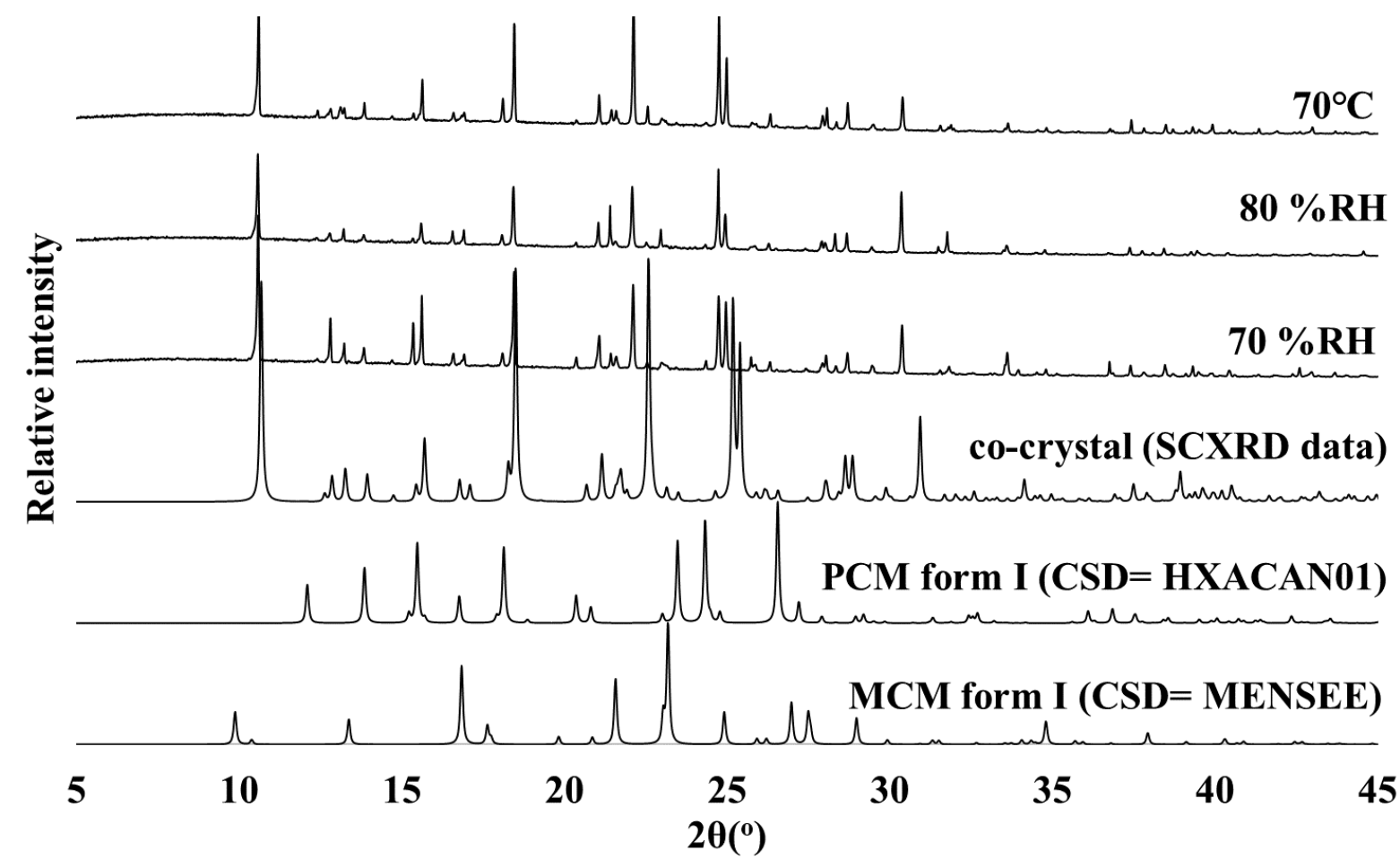

SI Figure 9. The PXRD traces of the co-crystal monitored after six weeks of being held in a chamber kept at $70^{\circ} \mathrm{C}$ and a chamber set a relative humidity of both 70 and $80 \%$. 


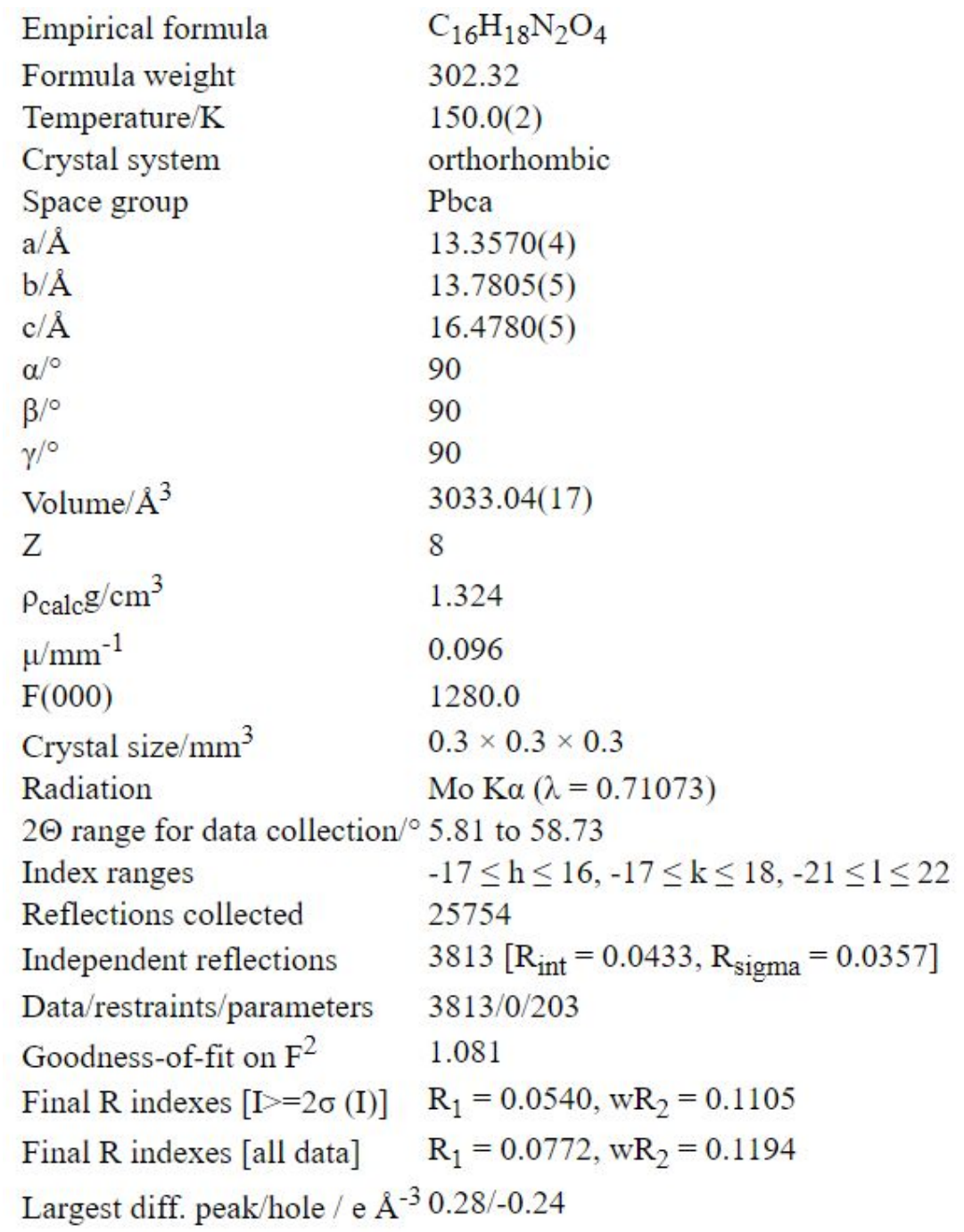

SI Figure 10. Crystallographic data for the MCM PCM co-crystal. 


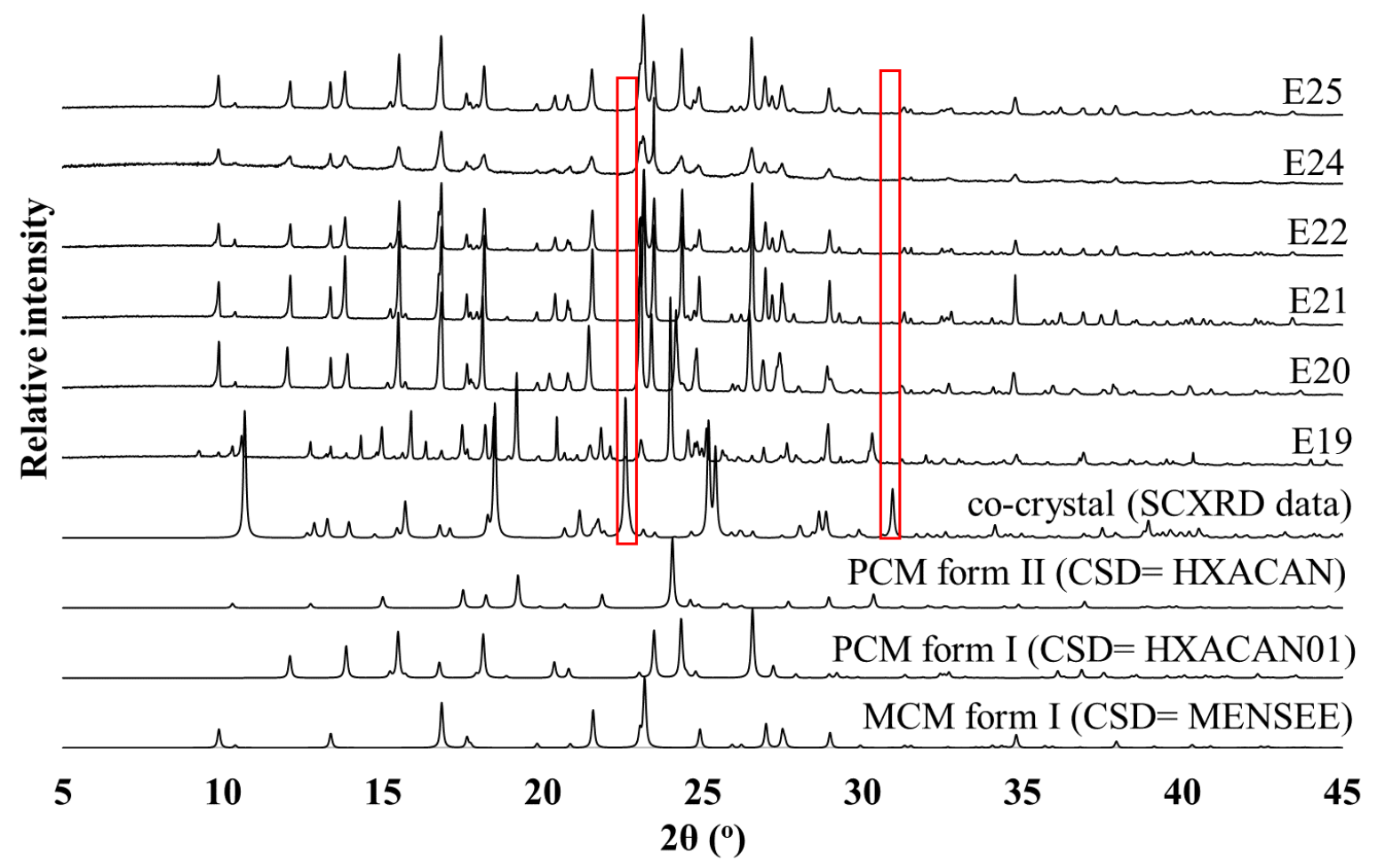

SI Figure 11. The PXRD traces obtained for a variety of non-seeded crystallizations, from experiments E19-E25 in SI Table 3. The red boxes highlight $2 \theta$ peaks from the co-crystal XRD trace that are missing in all other crystallization method traces.

a)

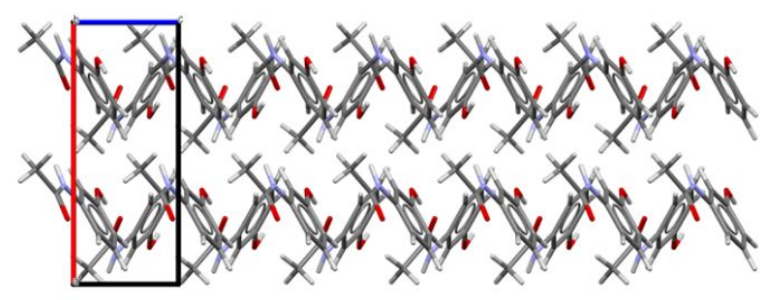

b)

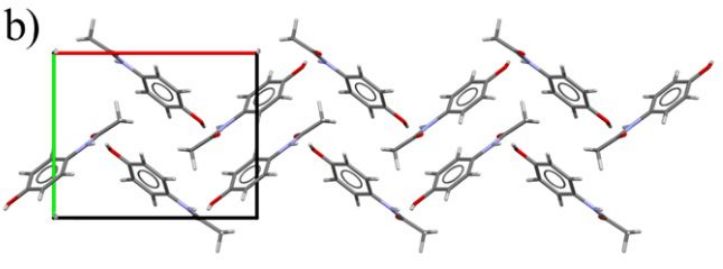

SI Figure 12. The packing arrangement of a) MCM form I (CSD= MENSEE), viewed along the $\mathrm{b}$ axis and $\mathrm{b}$ ) PCM form I (HXACAN01) viewed along the $\mathrm{c}$ axis. 


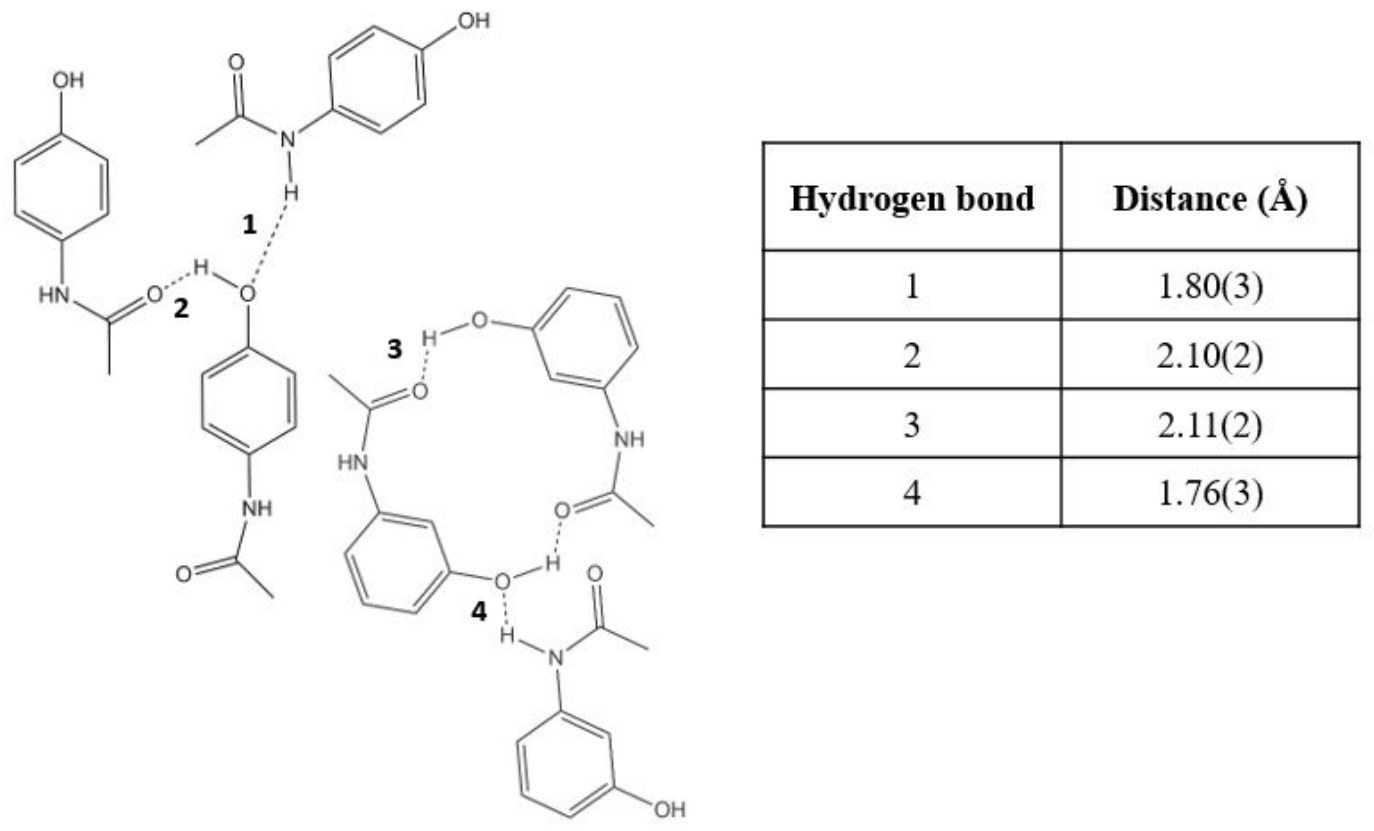

SI Figure 13. The intermolecular interactions between molecules of MCM and PCM in the co-crystal and their lengths.

Note that PCM is hydrogen bonded to other PCM molecules by two moderate hydrogen bonds: 1) $\mathrm{N}-\mathrm{H}$...O-C of length $1.80(3) \AA$; 2) $\mathrm{C}=\mathrm{O}$... H-O of length $2.10(2) \AA$. Likewise, $\mathrm{MCM}$ is hydrogen bonded to other MCM molecules by two moderate hydrogen bonds: 1) $\mathrm{C}=\mathrm{O}$...H-O of length $2.11(2)$; 2) N-H...O-C of length 1.76(3). There are no hydrogen bonds present between the molecules of MCM and PCM. 
PCM-form I \& II<smiles>CC(=O)Nc1ccc(C(NC(C)=O)N(COC(C)=O)c2ccc(O)cc2)cc1</smiles>

MCM-form I

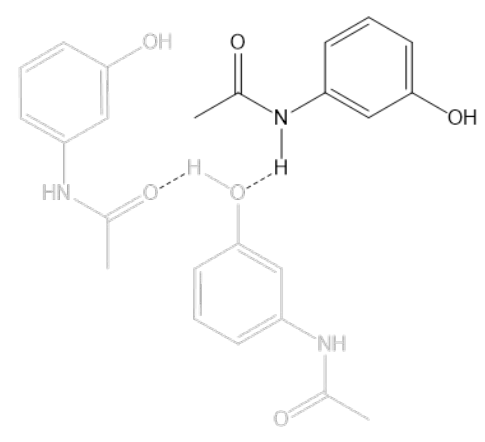

PCM in the co-crystal

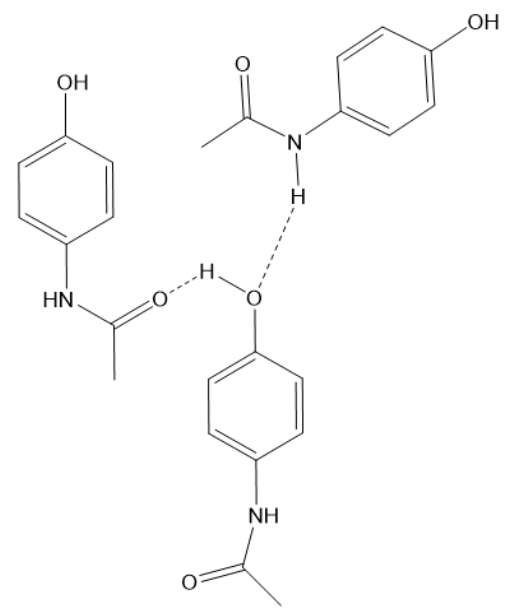

MCM in the co-crystal

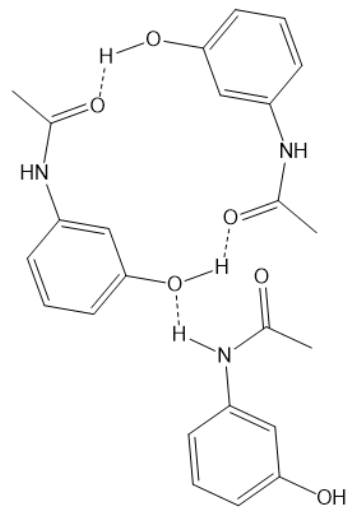

MCM-form II
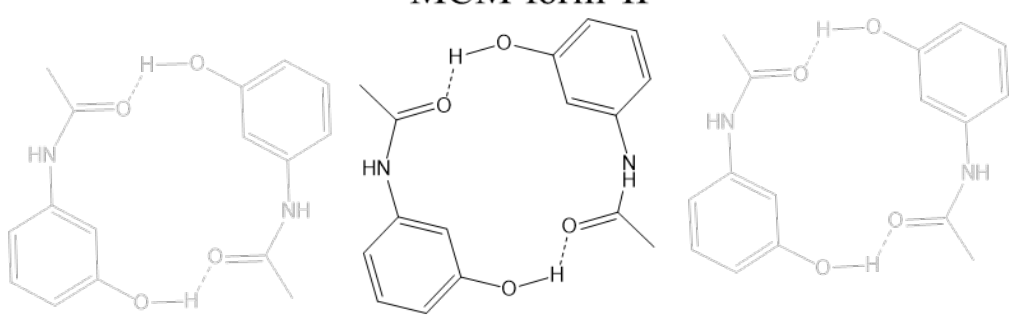

SI Figure 14. A comparison of the hydrogen bonding network in 1) the PCM molecule observed in the co-crystal and the PCM molecules in PCM form I and II; 2) the MCM molecule observed in the co-crystal and the MCM molecules in MCM form I and II. Parts of the hydrogen bonding network that are observed in both the co-crystal and single component are highlighted in black. 


\section{SI experimental}

Gravimetric methods

Gravimetric analysis was used to calculate a materials solubility at a fixed temperature. All measurements were prepared in a $20 \mathrm{~mL}$ glass vial that contained $10 \mathrm{~mL}$ of the binary solvent system. An excess of MCM and PCM (1:1) was added to the vial until the solution was vastly over saturated and a suspension was created. The glass vial was sealed and put into the Polar Bear crystalliser at a set temperature and was stirred for over 12 hours at $700 \mathrm{rpm}$. After 12 hours, the stirring was turned off and the reaming solid suspended in solution was left to settle at the bottom of the vial, leaving a clear solution at the top. $1 \mathrm{~mL}$ aliquots of the clear solution were taken. The aliquots were placed into previously weighed empty vials by a syringe fitted with a filter, that ensured no solid was present. The vials containing the solution aliquots were left to evaporate. Upon complete evaporation the vials were weighed and the concentration of sample dissolved in the $1 \mathrm{~mL}$ aliquots was calculated at the set temperature.

\section{Observational methods}

$3 \mathrm{~mL}$ of the binary solvent system was placed into a $20 \mathrm{~mL}$ glass vial. The sealed glass vial was put into the Polar Bear crystalliser and left for $1 \mathrm{~h}$ to equilibrate at $4^{\circ} \mathrm{C}$ at $700 \mathrm{rpm}$. Small sample increments of known mass were added to the glass vial which was subject to a slow heating profile of $0.2{ }^{\circ} \mathrm{C} / \mathrm{min}$ and a stirring rate of $700 \mathrm{rpm}$. The temperature at which all sample was dissolved from the first increment was noted. This value was plotted against the known mass increment to afford the first point on the solubility curve. This method was repeated to gain additional points on the solubility curve until the desired end temperature was reached.

\section{Cooling crystallization}

A Cambridge Reactor Design Polar Bear Plus crystallizer was used to implement batch cooling crystallizations. The crystallizer enabled programmable heating and cooling profiles and magnetic bottom stirring rates. A solution that contained MCM and PCM (1:1) in a binary solvent system of IPA and water (4:6) was placed into a sealed glass vial. The concentration of solution was saturated at $40^{\circ} \mathrm{C}$ and was based on a solubility curve of MCM and PCM (1:1) in IPA and water (4:6). The temperature profile from Figure 3 in the manuscript was followed with the exception of seed addition at $30^{\circ} \mathrm{C}$. The crystallisation was left for 72 hours at $4^{\circ} \mathrm{C}$. The product gained from crystallization was filtered and dried at room temperature before analysis.

\section{Mechano-chemical crystallization (grinding)}

Neat mechano-chemical crystallizations were carried out manually with a pestle and mortar. A 1:1 mixture of MCM and PCM were ground.

Liquid assisted grinding (LAG)

Neat mechano-chemical crystallizations were carried out manually with a pestle and mortar with 2 drops of solvent. A 1:1 mixture of MCM and PCM were ground.

\section{Solution-mediated crystallization (slurry)}


A solution that contained excess amounts of MCM and PCM (1:1) in a binary solvent system of IPA and water (4:6) was left at $40^{\circ} \mathrm{C}$ and stirred at $700 \mathrm{rpm}$ for 2 weeks. The product gained from crystallisation was filtered and dried at room temperature before analysis.

\section{Evaporative crystallization}

$3 \mathrm{~mL}$ of solution that contained MCM and PCM (1:1) in a binary solvent system of IPA and water (4:6) was placed into a $7 \mathrm{~mL}$ glass vial. Sonication in a temperature controlled water bath set at $40^{\circ} \mathrm{C}$ was used to aid dissolution. After dissolution, the vial lids were pierced with ten small holes to ensure slow evaporation of solvent. The vials were left to crystallise in hotplate set at $40^{\circ} \mathrm{C}$.

\section{Vapour diffusion crystallization}

A saturated solution of MCM and PCM (1:1) in a binary solvent system of IPA and water (4:6) was prepared. The solution was placed into a $1 \mathrm{~mL}$ glass vial. Sonication in a temperature controlled water bath set at $40^{\circ} \mathrm{C}$ was used to aid dissolution. The $1 \mathrm{~mL}$ glass vial was left unsealed and placed inside a larger sealed glass vial. The larger glass vial contained enough anti-solvent (hexane) to ensure that the solvent level was raised above the level of the solvent in the $1 \mathrm{~mL}$ vial but did not enter it. The large glass vial was sealed and was left to crystallise in a hotplate set at $40^{\circ} \mathrm{C}$. The product gained from crystallisation was filtered and dried at room temperature before analysis. 